\title{
Les ouvriers nantais dans la révolution française.
}

Une proposition pour l'étude de la participation des ouvriers à la révolution française : le sort des organisations ouvrières

The Workers in Nantes during the French Revolution. A proposal for a study of the participation of workers during the French Revolution: the fate of worker's organizations

\section{Samuel Guicheteau}

\section{(2) OpenEdition}

Journals

\section{Édition électronique}

URL : https://journals.openedition.org/ahrf/10554

DOI : $10.4000 /$ ahrf. 10554

ISSN : 1952-403X

Éditeur :

Armand Colin, Société des études robespierristes

\section{Édition imprimée}

Date de publication : 1 avril 2009

Pagination : 5-28

ISBN : 978-2-200-92558-1

ISSN : 0003-4436

Référence électronique

Samuel Guicheteau, "Les ouvriers nantais dans la révolution française. ", Annales historiques de la Révolution française [En ligne], 356 | avril-juin 2009, mis en ligne le 01 juin 2012, consulté le 24 avril 2022. URL : http://journals.openedition.org/ahrf/10554; DOI : https://doi.org/10.4000/ahrf.10554 


\title{
LES OUVRIERS NANTAIS \\ DANS LA RÉVOLUTION FRANÇAISE. UNE PROPOSITION POUR L'ÉTUDE DE LA PARTICIPATION DES OUVRIERS À LA RÉVOLUTION FRANÇAISE : LE SORT DES ORGANISATIONS OUVRIÈRES
}

\begin{abstract}
À la fin du XVIII ${ }^{e}$ siècle, Nantes est une grande ville industrielle et ouvrière. Tout en offrant une forte diversité, le monde ouvrier présente des traits communs tant sur le plan de la condition socio-économique que sur celui-ci de l'identité socio-culturelle. Dans la seconde moitié du XVIII ${ }^{e}$ siècle, les conflits du travail se multiplient en réaction aux exigences nouvelles que porte l'industrialisation. Dans ce contexte, les organisations ouvrières se renforcent, si bien que le problème de leur reconnaissance se pose d'autant plus fortement durant la Révolution. En effet, à Nantes comme ailleurs, les ouvriers interprètent l'abolition des privilèges et la Déclaration des droits de l'homme comme une liquidation des privilèges corporatifs et de la police du travail, donc comme une légalisation de leurs organisations. Après une période de tolérance, la loi Le Chapelier réaffirme nettement leur interdiction. Par la suite, les ouvriers peuvent néanmoins entretenir leur expérience révolutionnaire dans leurs organisations, si bien que celles-ci connaissent des évolutions.
\end{abstract}

Mots-clés : ouvriers, compagnonnages, Nantes.

À la fin du XVIII ${ }^{\mathrm{e}}$ siècle, Nantes est une grande ville industrielle et ouvrière. Puissante, l'industrie nantaise se compose de multiples activités et est engagée dans une profonde modernisation. Plusieurs activités sont concentrées. De plus, la production dispersée ne reste pas en marge de l'industrialisation, définie comme un processus de mutation générale. Le 
monde ouvrier nantais est très important. S'il reste inséré par plusieurs aspects dans l'ensemble du peuple et s'il se caractérise par une forte diversité, liée à la multiplicité des activités, à la pluralité des cadres de travail et à la hiérarchie des niveaux de qualification, il présente aussi des traits communs tant sur le plan de la condition socio-économique que sur celui de l'identité socio-culturelle. Surtout la plupart des ouvriers nantais partagent une identité forgée au travail ${ }^{1}$ : si elle n'engendre pas une conscience collective - le métier restant essentiel - elle constitue néanmoins un élément fondamental de la définition du monde ouvrier car elle est façonnée par les ouvriers eux-mêmes.

Il reste pourtant très difficile de répondre à « l'impertinente question : qu'est-ce qu'un ouvrier? $»^{2}$. En effet, la seule mention du métier masque la condition sociale exacte des individus. Le terme " ouvrier», qui peut désigner l'ensemble des « gens mécaniques », concourt aussi à cette imprécision. Certaines sources - notamment policières et judiciaires - livrent néanmoins la condition sociale. L'appréhension du monde ouvrier est, en outre, rendue particulièrement délicate par l'entrecroisement des aspects juridiques et sociaux jusqu'à l'abolition des corporations, et par l'ampleur des évolutions en cours. Pour nous, il se compose donc des salariés des manufactures et des ateliers, ainsi que des chambrelans ${ }^{3}$ : c'est en ce sens que nous emploierons nous-même le terme « ouvrier ». De plus, sans les confondre, de nombreux maîtres deviennent façonniers et travaillent parfois isolément. Sans avoir jamais été délaissée $e^{4}$ l'étude du monde ouvrier durant la Révolution connaît un regain d'intérêt ${ }^{5}$.

(1) Voir plus bas la présentation de ses fondements.

(2) Nous reprenons le titre du célèbre placard de 1793 intitulé Réponse à l'impertinente question: mais qu'est-ce qu'un sans-culotte?

(3) Les allers-retours d'une situation à l'autre sont fréquents (Michael SONENSCHER, Work and Wages. Natural Law, Politics and the Eighteenth-Century French Trades, Cambridge, CUP, 1989).

(4) Il est impossible de citer tous les travaux qui traitent du monde ouvrier. Citons néanmoins d'emblée Dominique Godineau, Citoyennes tricoteuses. Les femmes du peuple à Paris pendant la Révolution française, Aix-en-Provence, Alinéa, 1988, et Haim Burstin, Une révolution à l'œuvre. Le faubourg Saint-Marcel (1789-1794), Seyssel, Champ Vallon, 2005. Par ailleurs, l'ouvrage de W. Sewell, Gens de métier et révolutions. Le langage du travail de l'Ancien Régime à 1848, Paris, Aubier-Montaigne, 1983 (1980), a suscité un important débat au sujet tant de la participation des ouvriers à la Révolution que de leur identité sociale et culturelle ; nous renvoyons à l'éclairante présentation qu'en donne S. KAPLAN dans La fin des corporations, Paris, Fayard, 2001, p. 580 et suiv., non sans préciser que notre approche de l'identité des ouvriers se focalise sur le travail, creuset de pratiques et de valeurs fondamentales.

(5) Rendant compte du livre de Steven Kaplan, op. cit., Philippe Minard indique que cet ouvrage " souligne combien nous manquons d'études précises sur la façon dont le monde artisanal et ouvrier a vécu la Révolution» (Annales. Histoire, sciences sociales, 2006-4, p. 951). Jean-Pierre Jessenne déplore encore notre méconnaissance des milieux populaires («L'histoire sociale de la 
Pour comprendre la participation des ouvriers à la Révolution française, il nous semble nécessaire de saisir les problèmes auxquels ils sont confrontés à la fin de l'Ancien Régime et les aspirations qu'ils nourrissent, car ils les investissent sans doute dans la Révolution. De même, leur participation à celle-ci est, pour partie, façonnée par leurs pratiques et leurs valeurs. À la suite d'Edward Thompson qui évoquait la manière dont les ouvriers anglais avaient vécu la révolution industrielle, nous suggérons de ne pas considérer les ouvriers nantais comme " un matériau humain brut $»^{6}$ lorsque la Révolution éclate. Cette proposition ne signifie pas qu'ils traversent la Révolution aveugles à tout ce qui ne les concerne pas directement, mais que leur expérience révolutionnaire se nourrit tout autant de leur identité que de leur implication dans la Révolution. Nous proposons ici de mettre en œuvre cette approche à travers la question des organisations ouvrières ${ }^{7}$.

\section{La montée des tensions dans la seconde moitié du XVIII ${ }^{\mathrm{e}}$ siècle}

Pour saisir le sort des organisations ouvrières durant la Révolution française, il faut d'abord présenter leurs caractéristiques et l'évolution qu'elles connaissent dès la fin de l'Ancien Régime.

\section{Une incessante agitation}

Dans la seconde moitié du XVIII ${ }^{e}$ siècle, le monde ouvrier nantais semble en permanence agité. Cette agitation est entretenue par les organisations ouvrières ${ }^{8}$, qui s'efforcent de contrôler le marché du travail en assurant le placement de leurs membres et en empêchant les maîtres

(Suite de la note 5 page 4).

Révolution française : entre doute et renouvellement », dans Jean-Clément MARTIN (éd.), La Révolution à l'œuvre. Perspectives actuelles dans l'histoire de la Révolution française, actes du colloque de Paris, janvier 2004, Rennes, PUR, 2005, p. 35). Dans un récent ouvrage, Jeff Horn propose un regard stimulant sur la participation des ouvriers à la Révolution (The Path not Taken. French Industrialization in the Age of Revolution, 1750-1830, Cambridge, MITP, 2006) ; précisons que nous n'avons pas relevé à Nantes d'acte de luddisme. (1963).

(6) Edward Thompson, La formation de la classe ouvrière anglaise, Paris, Seuil, 1988, p. 74

(7) Précisons bien que nous traitons ici des organisations d'ouvriers et que les corporations de maîtres n'entrent donc pas dans notre étude puisque, « faute de maîtrise, les compagnons [en] sont exclus » (Steven KaPLAN, Philippe Minard [éd.], La France, malade du corporatisme ? XVIII ${ }^{e}$-XXe siècles, Paris, Belin, 2004, p. 13).

(8) Les caractéristiques originales des organisations ouvrières du XVIII ${ }^{\mathrm{e}}$ siècle sont telles que leur définition ne peut être élaborée que progressivement (voir plus bas, en particulier la proposition avancée par $\mathrm{Ph}$. Minard). 
d'embaucher hors de leurs rangs. De cet effort témoigne la double interdiction édictée par le règlement royal de 1749 :

« Faisons [...] défenses à tous les compagnons et ouvriers de s'assembler en corps sous prétexte de confrairie ou autrement, de cabaler entre eux pour se placer les uns les autres chez des maitres ou pour en sortir, ni d'empescher [...] les maîtres de choisir eux-mêmes leurs ouvriers soit françois ou étrangers $»^{9}$.

L'enjeu salarial est évident. Le 16 novembre 1786, le tribunal de police rappelle aux tisserands l'obligation de détenir un livret compilant les certificats de congé et de respecter le préavis de huit jours avant le départ ${ }^{10}$. Or, ce rappel intervient dans une période où les compagnons semblent engagés dans un conflit salarial durable puisqu'en 1787 une descente de police vise leur auberge de prédilection et qu'en 1788 une ordonnance leur interdit de revendiquer une augmentation de salaire ${ }^{11}$.

Le placement compagnonnique apparaît dans de nombreux métiers. En 1761, un tailleur d'habits reconnaît que « ce sont les garçons du devoir qui le place dans les villes par où il passe $»^{12}$. En l'an VII, le rôleur des maréchaux tente de se dédouaner en présentant sa charge sous l'angle de la sociabilité : «Si un camarade arrivant me propose de boire une bouteille et me demande où il y a de l'ouvrage, je crois permis de lui enseigner, mais je ne l'y ai jamais conduit $»^{13}$. Pour s'assurer un monopole, les ouvriers s'efforcent aussi d'écarter les étrangers et de limiter le nombre d'apprentis. Les constructeurs de navires de l'avant-port de Paimbœuf se plaignent, en 1777, d'être "privé[s] du secours des ouvriers étrangers, parce que les ouvriers du païs leur deffendent de travailler $\rangle^{14}$. Confrontés à une coalition de l'ensemble des imprimeurs en 1787, les patrons d'une indiennerie dénoncent ces ouvriers qualifiés, «qui abusant de leur petit nombre et se refusant le plus qu'ils peuvent à former des élèves, tiennent les maîtres des manufacture dans leur dépendance presque à tous égards et surtout pour le taux du salaire $»^{15}$. Enfin, les portefaix sont organisés en

(9) Archives municipales de Nantes (ci-dessous AMN), FF 92, registre des lois, arrêt du conseil du roi du 2 janvier 1749, fo 36,11 janvier 1781 .

(10) Ibid., HH 174, ordonnance municipale.

(11) Ibid., FF 257, procès-verbal du commissaire Bar, 11 février 1787 ; HH 174, ordonnance municipale, 11 décembre 1788.

(12) Ibid., FF 257, interrogatoire de Charles Garnier, 27 juin 1761.

(13) Archives départementales de Loire-Atlantique (ci-dessous ADLA), L 2086, interrogatoire de Jean Louis Beauvais, 7 germinal an VII.

(14) Ibid., C 676, projet de règlement, juillet 1777.

(15) AMN, HH 34, plainte d'Orillard, Guillou et Metzener, 8 octobre 1787. 
plusieurs bandes qui dominent chacune un quai et prétendent détenir le monopole d'une marchandise ${ }^{16}$.

Certains compagnonnages sont parvenus à s'assurer le contrôle du marché du travail et résistent vigoureusement contre les tentatives des maîtres pour le leur arracher. Ainsi, le 31 juin 1775, face à la volonté de la corporation d'appliquer la législation sur le placement, les taillandiers désertent leurs ateliers ${ }^{17}$. Le compagnon Langevin la Bouche d'Or estime que les billets de congé sont un moyen pour les maîtres de les payer aux «prix les plus bas $»^{18}$. Ces derniers dénoncent depuis longtemps le monopole compagnonnique :

«Le chef de cette association se nomme le roulleur, c'est lui qui dispose à son gré aux compagnon ; il les place dans les boutique des maistres qui lui sont agréable, ceux qui ont le malheur de lui déplaire sont assurer de n'en point avoir. Si dans les boutiques que le rouleur protège, il n'y a point d'ouvrage, les garçons du devoir forcent plutôt les arrivans à sortir de la ville que de souffrir qu'ils entrent dans les boutiques de ceux qu'ils n'aiment pas [...] ils forcent les compagnons qui ne sont pas de leur association et qu'ils appellent les gaveaux à sortir de la ville et ne veullent pas souffrir qu'ils travaillent $»^{19}$.

En 1776 encore, après avoir sommé quatre taillandiers étrangers au compagnonnage "de batre aux cham », les compagnons assiègent leur boutique $^{20}$. Cependant, des accords peuvent aussi être passés entre patrons et ouvriers, comme en témoigne celui qui, en 1777, prévoit qu'en période de sous-activité le travail sera réparti entre les peintres faïenciers, afin qu'aucun ne soit renvoyé ${ }^{21}$.

Pour contrôler le marché du travail, les ouvriers n'hésitent donc pas à se montrer violents. Pour imposer leurs revendications, les portefaix interrompent une décharge commencée et s'opposent à ce que d'autres l'achèvent. En mars 1783, la revendication d'une augmentation générale de dix sols incite de nombreux ouvriers à cesser le travail à Paimbœuf et, sur leur menace, les chantiers navals sont désertés ${ }^{22}$. Les compagnonnages

(16) Ces prétentions sont condamnées par plusieurs règlements (ibid., FF 209) et apparaissent dans les multiples conflits qui éclatent sur les quais (FF 210).

(17) Ibid., FF 257, déclaration du procureur du roi, 2 juillet 1775.

(18) Ibid., interrogatoire de Jean Robin, 4 juillet 1775.

(19) Ibid., HH 166, supplique des maîtres taillandiers, 27 août 1764.

(20) Ibid., FF 257, procès-verbal du commissaire Fleurdepied, 10 mars 1776.

(21) ADLA, 4 E 2-431, minute de maître Briand, 22 décembre 1777.

(22) Ibid., C 676, lettre des capitaines dont les navires sont en radoub, 26 mars 1783. 
recourent à la pratique de l'interdit et leurs membres molestent les récalcitrants. L'interdit peut frapper un unique atelier, par exemple, en l'an VII, celui d'un maître maréchal qui a prétendu " qu'il ne falloit pas que les ouvriers gagnassent plus de douze francs $»^{23}$. Dans les années 1780, les cordonniers mènent un combat pour l'augmentation de leur salaire. En 1780 d'abord, "les compagnons s'attroupèrent [et] évacuèrent dix à douze des plus fortes boutiques », si bien qu'ils obtinrent satisfaction. "Fruit d'une cabale criminelle», cette augmentation est rapidement supprimée. En 1786, les compagnons reprennent l'offensive « en désertant et faisant déserter les boutiques des maîtres ». De plus, ceux-ci ne peuvent pas embaucher « les compagnons arrivant [car] ceux du lieu les ont fait passer de bout en leur donnant de l'argent pour continuer leur route $»^{24}$. Enfin, l'interdit peut également frapper toute la ville : Nantes est désertée par les cloutiers en $1769^{25}$, par les taillandiers en 1775. Parfois, les organisations ouvrières s'adressent aux autorités. Ainsi, le 7 septembre 1786, les compagnons cordonniers déposent une supplique au tribunal de police « afin d'obtenir une augmentation de leur salaire, persuadés qu'ils sont qu['ils] leur accorder[ont] cette grâce, ainsy qu[ils] l'[ont] fait aux ouvriers des différents états $»^{26}$.

La défense de la condition ouvrière mobilise une identité fondée sur l'exaltation de la qualification et l'attachement à l'autonomie. Les compagnonnages se présentent comme des écoles de qualification. Tel est l'argument avancé par un maître charpentier, ayant lui-même appris son métier sur le tour de France :

«Il est bon de vous expliquer, Monsieur, la différence qu'il y a d'un compagnon du devoir d'avec celui qu'on nomme renard : un compagnon du devoir est celui qui a fait preuve de sa probité devant ses camarades et de son savoir-faire. Pour y parvenir, il subi nombre d'examen pratique sur l'art du trait de charpente [...] Alors il est reçu compagnon du devoir et a part aux bienfaisances de cette société qui est qu'en voyageant [...] on lui donne des secours [et] de l'ouvrage. Ces secours donnent les moyens aux jeunes gens les plus pauvres de voyager et faire, comme on dit, son tour de France. Par conséquent, il peut devenir artiste en son genre $»^{27}$.

(23) Ibid., L 2086, plainte de Pasquier, 10 germinal an VII.

(24) AMN, HH 123, supplique des maîtres cordonniers, 16 septembre 1786.

(25) Ibid., HH 120, certificat des maitres cloutiers de Paimbœuf, 10 avril 1769.

(26) Ibid., HH 123.

(27) Ibid., I 2-C 7-D 4, lettre de Bousset, 6 août 1791 (citée dans Michael Sonenscher, Work and Wages, op. cit., p. 315). 
Par ailleurs, au-delà du degré réel de qualification, compte le sentiment de posséder un savoir-faire car il nourrit la conscience professionnelle ${ }^{28}$, en même temps - bien sûr - qu'il justifie la revendication. Ainsi, aussi ténu soit-il, le savoir-faire des portefaix est réel ${ }^{29}$. De plus, le monopole sur une marchandise particulière engendre des tours de main spécifiques. Les débardeurs invoquent ce savoir-faire pour récuser l'emploi de simples journaliers.

À la défense de la qualification s'articule celle de l'autonomie. Celle-ci s'épanouit notamment à travers l'élasticité du temps consacré au travail. En l'an VII, les maréchaux reprochent également à un maître d'avoir congédié deux ouvriers « qui, au lieu de travailler, sacrifioient leurs journées entierres à la ribotte », et de les avoir remplacés par un journalier ${ }^{30}$. L'autonomie nourrit également une inclination à la mobilité. Les changements d'atelier au sein d'une ville, voire les déplacements de ville en ville pour les ouvriers qualifiés sont souvent motivés par la recherche de meilleures conditions. Les compagnonnages favorisent la mobilité en vue de créer sur place une pénurie de main-d'œuvre favorable à de meilleurs salaires. Une organisation de graveurs d'indiennes apparaît même à travers la recommandation dont bénéficie l'un d'eux pour le patron d'une manufacture installée dans la ville qu'il rallie $^{31}$. Les compagnons taillandiers veillent à entretenir la rotation des ouvriers d'atelier en atelier ${ }^{32}$. Ainsi, l'agitation qui anime le monde ouvrier nantais tient non seulement à la défense de la condition ouvrière par de puissantes organisations, mais encore au-delà à l'identité même de ces ouvriers pour qui la qualification et l'autonomie sont des valeurs autant que des pratiques.

(28) Gérard Gayot et Philippe Minard écrivent que « la qualification fait l'objet de très forts investissements symboliques, elle cristallise l'identité au travail à travers le sens du métier et de l'honneur professionnel » (Les ouvriers qualifiés de l'industrie (XVI $\mathrm{e}-X X^{\mathrm{e}}$ siècles). Formation, emploi, migrations, actes du colloque de Roubaix, novembre 1999, Revue du Nord, hors-série n 15, 2001, p. 9).

(29) Voir plus généralement Dockers de la Méditerranée à la mer du Nord. Des quais et des hommes dans l'histoire, actes du colloque d'Aix-Marseille, mars 1999, Aix-en-Provence, Edisud, 1999. Nous rejoignons ici la définition que donne Michel Pigenet du savoir-faire comme une « combinaison de connaissances, d'expérience et d'habileté [qui] s'évalue aussi en puissance, aptitude à économiser ses efforts, endurance, vitesse et intuition du danger » ("Aux fondements d'une identité. Retour sur deux siècles de travail ouvrier », Historiens-Géographes, octobre 1995, p. 250).

(30) ADLA, L 2086, plainte de Pasquier et Huret, 2 germinal an VII.

(31) Ibid., L 2087, lettre de la mère Laignel à son fils, 26 brumaire an VII.

(32) AMN, HH 166, supplique des maîtres taillandiers, 27 août 1764. 


\section{L'exacerbation des conflits}

L'essor économique rend de plus en plus insupportables ces pratiques qui, nourrissant une agitation permanente, l'entravent. Les efforts entrepris pour les déraciner se heurtent toutefois à une résistance d'autant plus vigoureuse que les ouvriers en usent pour tirer parti de la croissance de l'activité. Les sempiternelles escarmouches qui troublent les quais se muent, en août 1775, en un grand conflit à propos des cargaisons américaines. Qu'une telle confrontation éclate au sujet de ces marchandises dont le trafic est au cœur de la croissance nantaise montre bien que l'essor rend de plus en plus inacceptables aux yeux des négociants les pratiques traditionnelles des portefaix. À la suite de ce grand conflit, une ordonnance exige de manière plus draconienne qu'auparavant qu'ils se consacrent au travail et renoncent aux multiples plaisirs qui les en détournent ${ }^{33}$. Vers 1780, le Commerce de Nantes réclame de plus en plus fortement la promulgation d'un règlement pour les chantiers navals de Paimbœuf où règne « parmi les ouvriers un désordre qui devient de plus en plus préjudiciable à la navigation et aux intérêts du commerce maritime $»^{34}$.

En 1787, une nouvelle indiennerie se consacre à la production d'indiennes de traite de médiocre qualité. L'impression est si simplifiée qu'elle peut être confiée à des ouvrières dépourvues de qualification. Face aux protestations des imprimeurs, les patrons précisent que le versement de faibles salaires est nécessaire pour soutenir la concurrence. Au-delà, ils dénoncent leurs « débauches qui souvent laissent les atelliers déserts des semaines entières », et indiquent qu'au contraire, les ouvrières, flanquées d'enfants, «ont besoin de travailler avec assiduité »" ${ }^{35}$. L'appel aux femmes vise donc à introduire une plus grande discipline. Lucide autant que cynique, le rédacteur de la plainte estime également qu'il est d'autant plus nécessaire « de réprimer les cabales de ces sortes de gens qui peuvent devenir plus dangereuses à mesure que les manufacture se multipliront ». Par ailleurs, la part en nature de la rémunération se trouve remise en cause. Ayant dû consentir en 1780 une augmentation de salaire aux compagnons, les maitres cordonniers les privent de «l'ordinaire [...], c'est-àdire la soupe et la viande les jours gras et la soupe seulement les jours maigres $»^{36}$. En 1786, la corporation des cloutiers tente de supprimer les

(33) Ibid., FF 209, ordonnance municipale, 20 février 1777.

(34) ADLA, C 676, adresse du Commerce de Nantes au parlement de Bretagne, 1778.

(35) AMN, HH 34, plainte d'Orillard, Guillou et Metzener, 8 octobre 1787.

(36) Ibid., HH 123, supplique des maîtres cordonniers, 16 septembre 1786. 
repas offerts par le maitre ${ }^{37}$, mais certains conservent l'usage de la « pitance » dans le dessein d'attirer les ouvriers ${ }^{38}$.

À l'inverse, les ouvriers s'appuient sur leurs pratiques traditionnelles pour tirer profit de la croissance de l'activité. Organisés en équipes indépendantes, les ouvriers de Paimbœuf n'hésitent pas à abandonner un chantier pour un autre, jouant habilement de la concurrence entre les constructeurs. Les ouvriers du bâtiment tentent de profiter de l'importante activité de construction. Celle-ci mobilise une main-d'œuvre issue de différentes provinces, comme l'indiquent en 1752 deux architectes confrontés à une coalition des tailleurs de pierre : "Pour faire tous ces ouvrages qui sont considérables, ils ont sans doute particulièrement [besoin] des tailleurs de pierre, et en effet ils en ont de différents païs ; [or] plusieurs particuliers aussy tailleurs de pierre de cette ville ont [...] formé la résolution de faire chasser tous les tailleurs de pierres [étrangers] $\gg^{39}$. Les Nantais leur reprochent de se contenter de bas salaires. Ménétra signale qu'en 1760, Nantes est défendue par les vitriers ${ }^{40}$. À mesure que le développement économique s'accentue, une contradiction se creuse donc entre les exigences nouvelles des maîtres et des négociants et la défense par les ouvriers de leurs pratiques.

Le renforcement de la police du travail accompagne le développement économique. Si ce durcissement vise à répondre aux nouvelles exigences portées par l'industrialisation, il provoque - de nouveau de manière contradictoire - une résistance qui aiguise les tensions. En 1749, le billet de congé est généralisé, et, en 1781 est institué le livret ouvrier, qui compile en fait les congés successifs : ces mesures visent à réduire la volatilité des ouvriers et à entraver leurs pratiques de lutte. À Nantes, le renforcement de la législation s'accompagne de tentatives pour créer des bureaux de placement, notamment en $1762^{41}$. En juin de cette année, les jurés tailleurs d'habits tentent de recenser les compagnons, mais ceux-ci se dérobent, l'un d'eux répondant même « que son nom étoit : je me fout de cela $\gg^{42}$. De plus, par calcul pour obtenir des ouvriers, par nostalgie de leur

(37) Ibid., HH 116, avis des maîtres cloutiers, 7 décembre 1786.

(38) Ibid., supplique des maîtres cloutiers, 18 mai 1789.

(39) Ibid., FF 259, plainte de Nicolas Rainard et Pierre Deprez, 13 mai 1752.

(40) Daniel Roche, Journal de ma vie. Jacques-Louis Ménétra, compagnon vitrier au XVIII siècle, Paris, Montalba, 1982, p. 63.

(41) Thierry Hamon, Les corporations en Bretagne au XVIII siècle. Étude statutaire et contentieuse, Université Rennes I, 1992, p. 261.

(42) AMN, HH 169, procès-verbal du commissaire Bar, 8-9 juin 1762. 
jeunesse ou par solidarité, étant eux-mêmes devenus façonniers ${ }^{43}$, certains maîtres refusent de souscrire aux nouvelles exigences de la police du travail. À la grande joie de ses ouvriers, un maitre couvreur déclare que s'ils lui présentaient des billets, « il en feroit des torches-culs $»^{44}$. Au début des années 1780, une offensive policière se produit. Elle se heurte à une forte résistance : irrités par la tentative des maîtres d'appliquer rigoureusement la police du placement ${ }^{45}$, les compagnons menuisiers se retirent de Nantes en $1781^{46}$. En outre, ce durcissement se traduit sur le plan idéologique par une exigence renforcée de soumission. Si, en 1777, le procureur du roi réitère à l'encontre des portefaix les interdictions usuelles, il déplore plus nettement qu'ils « se répandent souvent en injures et menaces contre ceux qui les emploient, ce qui est contraire au droit naturel qui veut que celui qui est payé soit subordonné à celui qui paie $\aleph^{47}$. En contrecoup, l'affirmation de la dignité ouvrière s'aiguise, à l'occasion de démarches juridiques, grâce à l'expression de la condition ouvrière en termes de droits et à l'emprunt d'un vocabulaire politique : ainsi, en 1768, les cloutiers dénoncent la volonté des maîtres de contrôler le placement comme une prétention qui « ne pouroit que dégénérer en tirannie $»^{48}$. La police du travail étant mise en œuvre au premier chef par les corporations, son sort se lie aux yeux des ouvriers à celui des jurandes elles-mêmes.

\section{Le renforcement des organisations ouvrières}

Selon Philippe Minard, « l'organisation ouvrière [d'Ancien Régime] procède moins d'une structure formelle que d'un ensemble de pratiques et de réflexes collectifs passés au rang des traditions $»^{49}$. Les organisations nantaises s'enracinent d'abord dans une intense sociabilité professionnelle. Née au travail, celle-ci s'épanouit au-delà, comme le suggère l'interdiction faite aux ouvriers d'un même métier de se réunir à plus de trois

(43) En 1787, les compagnons tisserands échappent à la police parce qu'un maître les a prévenus (voir plus haut).

(44) AMN, FF 255, procès-verbal du commissaire Bar, 5 septembre 1782.

(45) Le commissaire effectue une tournée de tous les ateliers de menuiserie, afin de vérifier l'application de la législation sur le placement (ibid., procès-verbal, du commissaire Bar, 25 août 1781).

(46) Ibid., HH 147, supplique des maîtres menuisiers, 12 septembre 1781.

(47) Ibid., FF 209, ordonnance municipale, 20 février 1777.

(48) Ibid., HH 120, supplique des compagnons cloutiers adressée au parlement de Bretagne, 15 décembre 1768. Sur ce point, outre Michael Sonenscher, Work and Wages, op. cit., voir Jean Nicolas, La rébellion française. Mouvements populaires et conscience sociale (1661-1789), Paris, Seuil, 2002

(49) Philippe Minard, Typographes des Lumières, Seyssel, Champ Vallon, 1989, p. 170. 
dans un cabaret. La répétition de cette interdiction signale sa vanité, ainsi que les multiples infractions constatées ${ }^{50}$. Les assemblées compagnonniques se tiennent dans l'auberge du Père, lieu de sociabilité autant que siège d'organisation. Ainsi, le 20 septembre 1767, la police arrête vingttrois cloutiers réunis à l'auberge du Lion d'argent ${ }^{51}$. Dans ces auberges, les compagnons disposent d'une chambre où ils cèlent leurs papiers : à l'auberge du Griffon, la police s'empare, le 24 juin 1781, de la cassette où les poêliers conservent leurs documents ${ }^{52}$. En outre, les organisations ouvrières prolongent la solidarité professionnelle. Si celle-ci n'est ni évidente ni systématique, elle est précisément confortée par sa prise en charge par l'organisation. Au quotidien, cette solidarité prend la forme de l'entraide. Lors de conflits, elle se révèle bien sûr décisive. Plus précisément, l'entraide bénéficie aux compagnons qui quittent les ateliers dont les maîtres rejettent les revendications, comme le dénoncent les maîtres cordonniers en 1786.

Les organisations ouvrières cristallisent les pratiques et les valeurs des ouvriers. «Échappée belle » hebdomadaire ${ }^{53}$, la saint lundi est consacrée par les compagnonnages comme jour de conduite ${ }^{54}$. De même, ceux-ci entretiennent l'idéal de qualification en codifiant le rapport symbolique aux outils. Le 8 août 1791, une rixe éclate entre des garçons menuisiers. Un ouvrier réagit violemment à l'intrusion de compagnons dans l'atelier où il travaille. Cette intrusion est justifiée par une coutume qu'expose le principal accusé : comme « il y avait une verlope sur l'étably, quoique personne ne fut dans la boutique, ce qui devait faire encourir l'amende au garçon, lui, interrogé, entra dans ladite boutique et frappa avec un marteau sur le fer de la verlope [...] tel est l'usage entre les garçons et [...] celui qui a fait l'oubli de sa verlope sur l'étably, paye une bouteille de vin, si c'est un Bon Enfant $»^{55}$.

(50) Voir les multiples procès-verbaux dressés au titre de la police des cabarets (AMN, FF 215).

(51) AMN, HH 120, procès-verbal du commissaire Fleurdepied.

(52) Ibid., FF 257, procès-verbal du commissaire Bar.

(53) En témoigne l'argument avancé par les maîtres cordonniers pour rejeter l'augmentation revendiquée par les compagnons en 1786 : «La preuve de la suffisance de leurs salaires se tire de leur inconduite, ils ne travaillent presque jamais les lundi et les mardi, ils remplissent les cabarets et les guinguettes ; s'ils manquoient d'argent, ils travailleroient plus assiduement ».

(54) La coutume veut que les compagnons escortent jusqu'à la lisière de la ville l'ouvrier qui s'en va.

(55) AMN, I 2-C 7-D 4, interrogatoire de Jean Barrat, 23 août 1791. Sur l'importance des coutumes, voir Robert DARNTON, "Une révolte d'ouvriers : le grand massacre des chats de la rue Saint-Séverin », dans Le grand massacre des chats. Attitudes et croyances dans l'ancienne France, Paris, R. Laffont, 1985, p. 75-100 ; Léonard RosENBAND, La fabrication du papier dans la France des Lumières. Les Montgolfier et leurs ouvriers, 1761-1815, Rennes, PUR, 2005 (2000). Rappelons qu'une interprétation trop littérale des textes utilisés par ces deux auteurs pour présenter les coutumes 
À la fin du XVIII ${ }^{\mathrm{e}}$ siècle, le renforcement des organisations ouvrières passe par leur implantation dans de nouveaux métiers ${ }^{56}$ et de nouvelles villes, par le développement consécutif de leurs réseaux et s'accompagne de leur institutionnalisation ${ }^{57}$. En 1779, le procureur du roi au parlement de Bretagne dénonce l'essor des compagnonnages et présente Nantes comme leur foyer dans la province ${ }^{58}$. Cependant, dès 1754, les serruriers défilent en ville « marchant deux à deux, à la suite de l'un de leur même compagnon qui, ayant une espèce de petite caisse [...], battoit une marche militaire $\aleph^{59}$. Nantes semble un point nodal des itinéraires compagnonniques : de nombreux ouvriers présents à Nantes portent des surnoms compagnonniques $^{60}$, sont issus de migrations lointaines ${ }^{61}$ et n'y sont que de passage ${ }^{62}$. Foyer compagnonnique, Nantes est le théâtre de rixes qui opposent des métiers rivaux, beaucoup plus que des obédiences hostiles. Leur multiplication témoigne de l'exacerbation de l'esprit de corps qui résulte sans doute de l'institutionnalisation des organisations. Celle-ci passe en effet par une élaboration plus poussée du récit des origines légendaires et par l'ostentation des signes d'appartenance. Ainsi, les compagnons arborent fièrement les cannes dont le port est interdit par le parlement en 1779. La saisie de cannes provoque d'ailleurs la fureur d'un tailleur de pierre en 1782, qui vient les réclamer au domicile d'un commissaire de police :

«Ce dernier, nous parlant d'un air furieux, nous a dit : comment $f \ldots$ monsieur, qu'avez-vous fait des cannes que vous avez saisi hier à mes camarades, jolis garçons, comme moi [...] ; nous avons été obligés de le pousser vivement hors de notre chambre $»^{63}$.

Suite de la note 55 page 13.

ouvrières a été critiquée (voir le compte rendu de l'ouvrage de L. RosenBAND par Ph. Minard dans les Annales historiques de la Révolution française, 2002-2, p. 228).

(56) Voir plus bas la tentative des maréchaux pour fonder, en 1791, un compagnonnage.

(57) Outre Émile CoORnAERT, Les compagnonnages en France, du Moyen-Âge à nos jours, Paris, éd. ouvrières, 1966, voir Cynthia Truant, The Rites of Labor. Brotherhoods of Compagnonnage in Old and New Regime France, Ithaca-Londres, CUP, 1984, ainsi que William SEWEL, op. cit.

(58) AMN, FF 92, registre des lois, fo 30, 18 février 1779.

(59) Ibid., FF 257, procès-verbal du commissaire Turpin, 29 mai 1754.

(60) Ibid., FF 69, fo 39 et 41, serment des garçons menuisiers et selliers, 28 avril et 5 mai 1750 (cette source est signalée par Cynthia TRUANT, The Rites of Labor, op. cit., p. 114).

(61) Outre les menuisiers et les selliers enregistrés en 1750, voir les taillandiers interrogés en 1775 et les ouvriers auxquels un livret est remis en l'an XII (ibid., I 2, registre des livrets).

(62) Les deux tiers des ouvriers cloutiers recensés le 20 décembre 1769 sont présentés comme « battant la semelle » (ibid., HH 120).

(63) Ibid., FF 257, procès-verbal du commissaire Albert, 23 septembre 1782. 
Le port de livrées complète la tenue du compagnon. En juillet 1772, les menuisiers dérobent celles des tailleurs de pierre, qui sollicitent l'aide des couvreurs, comme le précise l'un de ceux-ci : «Interrogé s'il est du devoir et si les garçons tailleurs de pierres qui étoient venus demander du secours en sont; répond qu'il en est, de même que lesdits tailleurs de pierres, et qu'on ne leur eu pas enlevé leurs livrées s'ils n'en avoient pas été $»^{64}$.

Les compagnonnages cultivent leur dimension religieuse. Dans la tradition confraternelle, ils s'occupent des enterrements : le 21 octobre 1777, le corps de Jean Baptiste Brié, compagnon menuisier décédé à l'Hôtel-Dieu, est « enlevé par les compagnons du Devoir » et conduit en procession à l'église Sainte-Croix ${ }^{65}$. La fête du saint patron est célébrée avec ferveur et ostentation, comme en témoigne l'autorisation sollicitée par les forgerons en 1791 :

"Quelques ouvriers forgerons [...] ont dit que le jeudy $1^{\text {er }}$ décembre arrive la fête de saint Eloi, leur patron; ils ont remontré que leur usage, pour solenniser cette fête, est de faire dire une messe et d'y assister, passé de quoi ils se réunissent chez leur mère ou ailleurs pour s'y amuser avec décence ; [...] le Bureau [...] permet aux dits ouvriers de faire dire leur messe à Saint-Nicolas et d'y assister, parce qu'ils s'y rendront seul à seul, sans violon, sans ruban ni aucune autre marque de corporations et parce qu'aussitôt après, ils se retireront de même chez la mère $»^{66}$.

Le 2 décembre 1781, des couvreurs sont surpris « à couper un pain pour distribuer aux compagnons à la messe $»^{67}$.

À la fin de l'Ancien Régime, les organisations ouvrières présentent à Nantes différents visages. S'arrogeant le titre de maîtres ${ }^{68}$, les portefaix s'inspirent du modèle corporatif pour conforter leurs prétentions monopolistiques. Ceux de la Fosse sont organisés dans une confrérie, dont la dissolution, au milieu du siècle, marque le début d'une répression accentuée contre les débardeurs ${ }^{69}$. Enfin, d'autres créent en 1782 une société pour la décharge des savons qui joint à cette visée monopolistique un

(64) Ibid., interrogatoire de Pierre Boucher, 14 juillet 1772.

(65) Ibid., GG 501, registre de sépultures de 1'Hôtel-Dieu.

(66) Ibid., 1-D-5, registre de délibérations de la municipalité de Nantes, f ${ }^{\circ}$ 99, 29 novembre 1791.

(67) Ibid., FF 257, procès-verbal du commissaire Bar.

(68) Les ordonnances successives leur interdisent de prendre ce titre (ibid., FF 209).

(69) Ibid., FF 210, sentence du tribunal de police, 6 février 1743. 
caractère confraternel et mutualiste ${ }^{70}$. Des organisations apparaissent dans les manufactures, comme on l'a vu pour les indienneurs. Au lendemain de l'émeute du 5 septembre 1791, la rumeur d'une conjuration des compagnons alarme les autorités. Un grand patron du textile avertit le maire « [qu'] on a dit à nos ouvriers que dans plusieurs fabriques [...], l'ancien de chaqune a reçu une pareille lettre $»^{71}$, c'est-à-dire une convocation à une assemblée. Ainsi, au-delà des compagnonnages strictement dits, il faut souligner la capacité d'organisation des ouvriers, qui n'aboutit pas toujours à une formalisation aussi poussée. Au-delà de ces différences, les organisations ouvrières nantaises de la fin du XVIII siècle sont toutes professionnelles et présentent les mêmes fondements : elles recouvrent la sociabilité et la solidarité, elles cristallisent les pratiques et les valeurs, en fait l'identité ouvrière elle-même. De plus, elles se renforcent pour deux raisons. D'une part, leur défense d'une identité remise en cause par l'essor économique suscite l'institutionnalisation des traditions qui les façonnent. D'autre part, face au renforcement de la police du travail, elles affirment la dignité ouvrière. Quelles que soient leurs formes ${ }^{72}$, elles peuvent donc bien être définies comme « la cabale institutionnalisée et permanente $»^{73}$. Leur importance et leur renforcement sont tels qu'il est indispensable de s'interroger sur leur sort durant la Révolution pour saisir la participation des ouvriers à celle-ci.

\section{Le sort des organisations ouvrières nantaises durant la Révolution française}

Importante et pour partie autonome et originale, la participation des ouvriers nantais à la Révolution française se développe sur plusieurs plans. Les ouvriers s'engagent dans les grands conflits politiques, religieux et militaires qui scandent la Révolution. Ils interviennent aussi pour des motifs sociaux, soit dans l'ensemble du peuple notamment lors des

(70) ADLA, 4 E 2-432, minute de maître Briand le Jeune, 2 septembre 1782.

(71) Ibid., L 2073, lettre de L. Saget à D. Kervégan, 23 novembre 1791.

(72) Une même organisation peut présenter différents visages, notamment dans sa quête d'une reconnaissance légale : confrérie sous l'Ancien Régime, société de secours mutuels ensuite, ni l'une ni l'autre ne pouvant cependant être réduites au rang de simple masque. Par ailleurs, certains métiers disposent de deux organisations, le compagnonnage accueillant les jeunes itinérants et la société de secours mutuels regroupant les sédentaires : tel est le cas à Nantes chez les cloutiers, la société de secours mutuels restant liée au compagnonnage pour le placement et la défense du salaire (ibid., 7 U 18, règlement de la société des cloutiers, an IX).

(73) Steven KaPLAN, « Réflexions sur la police du monde du travail, 1700-1815 », Revue historique, 1979-1, p. 58. 
émeutes $^{74}$, soit pour des motifs spécifiques. Ainsi, les sans-qualité protestent contre les privilèges corporatifs : à partir de février 1789, les tisserands forains violent délibérément le privilège d'accès prioritaire au marché aux fils détenu par les maîtres ${ }^{75}$. L'emploi est primordial : en 1790, les ouvriers de l'atelier municipal de charité protestent contre sa fermeture ${ }^{76}$. Enfin, la défense du salaire mobilise de nombreux ouvriers. La rumeur d'une baisse des salaires provoque une émeute à Paimbœuf le 23 août $1789^{77}$. En 1790, après avoir reçu une pétition des ouvriers maçons portant notamment sur les salaires, la municipalité interdit les assemblées d'ouvriers ${ }^{78}$.

Le problème de la reconnaissance des organisations ouvrières s'inscrit dans l'ensemble de cette participation des ouvriers à la Révolution française. Il se pose de manière d'autant plus forte que ces organisations se renforcent encore au début de la Révolution ${ }^{79}$, et de manière d'autant plus délicate qu'est en jeu l'extension au domaine social des droits révolutionnaires et que le malentendu de $1776^{80}$ se répète avec une plus grande acuité du fait de son insertion dans un contexte révolutionnaire. Précisément, le souvenir du « carnaval de Turgot » est si vif que les corporations survivent à la Nuit du 4 août, alors même que les ouvriers interprètent l'abolition des privilèges et la Déclaration des droits de l'homme, non seulement comme la liquidation des privilèges corporatifs qui leur ouvrirait la possibilité de s'installer, mais encore comme la suppression de la police du travail et, en conséquence, comme la reconnais-

(74) Alors qu'elles ont disparu depuis longtemps, des émeutes éclatent de nouveau en 1789 contre la disette, en 1790 contre l'octroi et en 1791 contre l'inflation. Les ouvriers y participent fortement, certains métiers jouant même un rôle décisif : les maçons en 1789 et les ouvriers du port en 1791.

(75) AMN, HH 174, procès-verbaux de police, février-mai 1789.

(76) ADLA, L 1440, procédure du tribunal de district, décembre 1790.

(77) Ibid., B 8745-VI, procédure du présidial.

(78) «Le bureau municipal s'étant fait rendre compte par des ouvriers mêmes du motif de leurs assemblées, il a vu qu'elles avaient pour objet d'engager leurs maîtres à faire avec eux des conventions [...] Mais des gens inconnus, vagabonds et sans aveu, ont pris de là occasion de s'attrouper, et même de s'insinuer adroitement et secrettement parmi les ouvriers » (AMN, F 7-C 1-D 1, 24 et 25 février 1790).

(79) Pour Paris en 1789-1791, voir Steven Kaplan, La fin des corporations, op. cit., et Haim Burstin, Une révolution à l'œuvre, op. cit. À Lyon se tient, le 25 octobre 1789, une assemblée générale des compagnons du Devoir (Maurice GARDEN, Lyon et les Lyonnais au XVIII siècle, Paris, Les Belles Lettres, 1970, p. 568).

(80) Du fait que les corporations mettent en œuvre la police du travail, leur suppression a pu être interprétée comme la disparition de celle-ci. Dès lors, la conviction d'une reconnaissance - du moins d'une tolérance - des organisations ouvrières a pu s'inscrire dans la « carnavalisation des rapports sociaux » provoquée par l'édit de Turgot (Steven KAPLAN, La fin des corporations, op. cit., p. 78). Précisons que l'édit de suppression des corporations n'a pas été appliqué en Bretagne. 
sance de leurs propres organisations ${ }^{81}$. D'une manière générale, les ouvriers intègrent les principes révolutionnaires à leurs combats ${ }^{82}$. Au printemps 1791, la montée des luttes ouvrières, l'aiguisement des tensions politiques et le rapprochement entre les organisations ouvrières et le mouvement démocratique ${ }^{83}$ provoquent une forte réaction contre le mouvement ouvrier. La loi Le Chapelier s'inscrit aussi dans l'ensemble de la politique des Feuillants pour arrêter la Révolution. Un grand débat a été ouvert au sujet de la portée de cette loi pour le processus révolutionnaire comme pour la société contemporaine ${ }^{84}$. En inscrivant nos recherches dans ce débat,

(81) Steven KAPLAN écrit que « pour les ouvriers, aucun des droits révolutionnaires n'était aussi important que la liberté de s'assembler » (ibid., p. 424).

(82) Par opportunisme ou par conviction? S'il est vrai qu'à l'occasion d'un conflit, les ouvriers peuvent user malicieusement de tous les arguments susceptibles de conférer une légitimité à leur action, il est aussi indéniable que les conflits favorisent la politisation. Pour notre part, la puissance du processus révolutionnaire d'une part, l'acuité des expériences réalisées par les ouvriers durant la Révolution, l'inscription de celles-ci dans une politisation esquissée dès la diffusion des Lumières et la création des tribunaux de prud'hommes à laquelle elles aboutissent d'autre part, nous incitent à penser que la conviction est profonde et durable.

(83) Outre Steven Kaplan, La fin des corporations, op. cit., voir Raymonde MonNier, L'espace public démocratique. Essai sur l'opinion publique à Paris de la Révolution au Directoire, Paris, Kimé, 1994.

(84) D’abord, pour le processus révolutionnaire. Steven Kaplan estime que les conflits entre maîtres et ouvriers ont atteint en 1791 une telle acuité que leur union dans le mouvement populaire en 1792 est improbable, en dépit du poids de la lutte pour les subsistances et de la mobilisation pour la défense nationale. Il avance donc une nouvelle interprétation de la sans-culotterie : celle-ci « fit office d'instrument de contrôle social postcorporatif ; l'ordre du jour des sans-culottes contribua à prévenir, déplacer et discréditer tout conflit sur le marché du travail [...] les chefs fabriquèrent une fiction [...] d'égalité fondée sur les méthodes réglementaires de l'Ancien Régime, rebaptisées en termes révolutionnaires ; les valeurs fondamentales des sans-culottes - patrie, famille, travail - interdisaient le genre d'anarchie qui avait fait de la première moitié de 1791 une période si pénible » $(\mathrm{La}$ fin des corporations, op. cit., p. 581). Steven Kaplan précise qu'il entend ainsi ouvrir un débat. De même, Philippe Minard écrit que « l'escamotage du social [provoqué par la loi Le Chapelier] a ouvert la voie à la construction de la catégorie socio-politique factice de "peuple", unifiée autour des thèmes du patriotisme et de la lutte pour les subsistances »; il précise ensuite que « le ressurgissement du social, au cours du processus révolutionnaire, sous la forme politique du "populaire", peut expliquer comment est née l'idéologie sans-culotte de l'an II, qui repose sur la reconstruction mythifiée de relations sociales harmonieuses au travail » («Le métier sans institution : les lois d'Allarde-Le Chapelier de 1791 et leur impact au début du XIX ${ }^{e}$ siècle », dans Steven KaPLAN, Philippe Minard [éd.], La France, malade du corporatisme?, op. cit., p. 86). À partir du cas de Lyon, Alain Cottereau critique, de manière virulente, tant ce qu'il appelle la «mythologie soboulienne » de la sans-culotterie qui accrédite l'idée d'un soutien ouvrier aux révolutionnaires radicaux, que l'historiographie « ouvriériste » qui occulte l'exécution des principaux chefs des canuts lors de la répression terroriste ( La désincorporation des métiers et leur transformation en "publics intermédiaires": Lyon et Elbeuf, 1790-1815 », dans Steven Kaplan, Philippe Minard [éd.], La France, malade du corporatisme?, op. cit., p. 125). Précisons que citer n'est pas adhérer.

Ensuite, pour la société contemporaine. Pour Steven Kaplan et Philippe Minard, la radicalisation de l'individualisme libéral par la loi Le Chapelier provoque un escamotage durable du social qui façonne le mouvement ouvrier français, dans son idéologie comme dans ses structures, et au-delà les relations 
nous nous efforcerons de mettre en lumière l'expérience révolutionnaire des ouvriers.

\section{Des hésitations au rappel de l'interdiction}

La Déclaration des droits de l'homme semble bien être interprétée, à Nantes aussi, comme une reconnaissance des organisations ouvrières. Ainsi, le 7 août 1790, un commissaire de police répond à la sollicitation d'un groupe de portefaix, organisation qu'auparavant il combattait. L'incipit de son procès-verbal témoigne d'un complet renversement de l'attitude de la police : "Nous, François Fleurdepied, commissaire de police de la ville et communauté de Nantes, rapportons qu'ayant été requis par les nommés Agaisse [et divers noms] portefaix et porteurs de blé de la Poterne, les deux premier prévaux de la frérie $»^{85}$. Or, ces débardeurs attendent de lui qu'il les appuie dans la défense de leur monopole de porteurs de blé, prétention également condamnée par les autorités jusqu'alors : ils expliquent clairement au commissaire que « de tous les tems, eux seulles avoit le droit de faire les décharges des grains $»^{86}$. La Révolution semble donc comprise par ces portefaix comme la satisfaction de l'ensemble de leurs revendications, non seulement la reconnaissance de leur organisation, mais encore celle de leur monopole. La municipalité elle-même se montre tolérante. Il semble qu'elle ait reçu les ouvriers de plusieurs métiers venus, par patriotisme, prêter le serment civique $^{87}$. Alors que l'aristocratie ne désarme pas et que la crise religieuse s'aggrave, peut-être la municipalitén'entend-elle se priver d'aucun soutien ${ }^{88}$. Arrêté en novembre 1791, pour avoir, selon les autorités effrayées par

(Suite de la note 84 page 18).

sociales («Le corporatisme, idées et pratiques : les enjeux d'un débat incessant », dans La France, malade du corporatisme?, op. cit., p. 5-31). Cependant, Alain Cottereau souligne les efforts importants réalisés par les différents acteurs sociaux et politiques pour intégrer les principes révolutionnaires au domaine social, efforts qui aboutissent à la création du tribunal des prud'hommes de Lyon en 1806 («La désincorporation... », art. cit. ; précisons qu'à Nantes, un tel tribunal ne sera fondé qu'en 1841).

(85) AMN, FF 210.

(86) Au nom de ce monopole sur le blé, ces portefaix cherchent en fait à pénétrer sur le territoire d'un groupe rival.

(87) Si le registre des délibérations de la municipalité ne signale que le serment des garçons perruquiers le 28 février 1791 (AMN, 1-D-3, fo 195), Camille Mellinet écrit qu'en 1790, « le serment à la constitution était une solennité pour tous les corps d'état » (La Commune et la Milice de Nantes, Nantes, Mellinet, t. 6, 1841, p. 172 ; il s'agit bien des salariés).

(88) Parce qu'elle redoute, en 1791, la traduction électorale de la crise religieuse dans les campagnes, la municipalité nantaise s'efforce d'accroître le poids électoral de la ville en diminuant fortement l'estimation de la journée de travail, donc le niveau du cens, si bien que le nombre d'actifs à Nantes passe de 5 à 11000 (Yvon Le GaLl, Les consultations générales en Loire-Inférieure (1789-an VII), Université de Nantes, 1976, p. 145). 
l'émeute du 5 septembre précédent, participé à une conjuration visant à relancer celle-ci, en fait pour avoir sollicité, de la part des compagnonnages déjà constitués, l'approbation de son dessein d'en créer un chez les maréchaux, Jean Potel explique « que voyant que l'on tollérait les sociétés des autres métiers, ils avaient cru pouvoir les imiter et qu'ils seraient égallement tollérés ${ }^{89}$. Sans doute un relâchement de la police du travail accompagnet-il cette relative tolérance envers les organisations.

Comment expliquer un tel revirement par rapport à l'Ancien Régime ? D'une part, l'ensemble des acteurs, au-delà même des ouvriers, peut penser que les principes révolutionnaires s'appliquent au domaine social, notamment la liberté d'association. D'autre part, les ouvriers soulignent la concordance entre leurs pratiques traditionnelles et les valeurs révolutionnaires. Ainsi, le maréchal Jean Potel présente la réunion prévue des différents compagnonnages comme une fraternisation. Un maître charpentier, qui prend la défense de deux compagnons accusés d'avoir molesté un ouvrier étranger au compagnonnage, explique que celui-ci est une école non seulement de qualification mais encore de vertu. À «la probité » individuelle répond la «bienfaisance » collective. Dès lors, la fraternité lui apparaît comme le prolongement révolutionnaire de la solidarité ouvrière traditionnelle :

« Ayant moi-même fait le tour de France à pied, j'ai eu occasion de connaître les compagnons charpentiers du devoir et j'ai admiré la fraternité qui règne entre eux et les secours mutuels qu'ils se donnent réciproquement. Je les ai toujours reconnu en général pour de bons citoyens $»^{90}$.

Une telle concordance de valeurs ôte à ses yeux tout crédit à la rumeur d'une suppression des compagnonnages colportée par les ouvriers qui leur sont étrangers.

La loi Le Chapelier (14 juin 1791) marque un tournant. Si une délibération municipale du 27 mai 1791 précise que les confréries professionnelles ne sont pas frappées par la dissolution des corporations (prononcée en mars par le décret d'Allarde) et peuvent continuer à organiser des processions ${ }^{91}$, les

(89) À cette date, cette tolérance n'est bien sûr plus de mise : l'édile municipal qui l'interroge rétorque « qu'il a tort d'alléguer cette prétendue tollérance qui n'a jamais eu lieu puisqu'au contraire la police a toujours fait ce qui était en elle pour empêcher ces sortes d'associations » et souligne «que de tout temps elles ont été défendues sous des peines par les lois et règlemens de police et que surtout depuis la nouvelle constitution elles sont expressément prohibées » (ADLA, L 2073, 25 novembre 1791).

(90) AMN, I 2-C 7-D 4, lettre de Bousset, 6 août 1791.

(91) Ibid., 1-D-4, fo 143. 
ouvriers menuisiers n'obtiennent pas l'autorisation de célébrer une messe et de s'assembler le 26 juillet ${ }^{92}$. En effet, au lendemain de l'adoption de la loi Le Chapelier, les autorités engagent une offensive contre les compagnonnages. Le 21 juillet, un tapissier est expulsé de Nantes pour avoir interdit un atelier ${ }^{93}$. Deux rixes banales qui éclatent quelques jours plus tard parmi les charpentiers, puis les menuisiers ${ }^{94}$, sont présentées comme les signes d'une offensive compagnonnique. Le procureur de la commune fustige « les excès que produit le nouveau régime de compagnonnage que les ouvriers de tous les états veulent établir au mépris des loix $\rangle^{95}$. Néanmoins, l'émeute populaire du 5 septembre et la conjuration compagnonnique qui la suit ${ }^{96}$ contraignent les autorités à renouer, provisoirement, avec une certaine tolérance : les forgerons sont autorisés le 29 novembre 1791 à célébrer la saint-Éloi pourvu qu'ils le fassent sans ostentation ${ }^{97}$.

Toutefois, dès le 31 janvier 1792, le procureur de la commune déplore qu' « il existe encore parmi les ouvriers de vieilles dénominations : des compagnons et garçons dits du devoir s'assemblent chez des mères, des grades se distribuent, des taxes se perçoivent, et des conduites tumultueuses terminées ordinairement par des rixes mortelles [...] se font tous les jours ${ }^{98}$. En juin 1792, les réjouissances des forgerons et des maréchaux sont interrompues par la police. Les premiers protestent prudemment de leur loyauté politique :

«Messieurs, qu'a-t-on à nous reprocher? nous sommes tous bons citoyens ; même il y en a dans notre corps qui sont enrôllés dans la garde nationale, et nous offrons nos services à la patrie toutes fois et quant elle aura besoin de nous; si nous avons porté quelques rubans suspects à la nouvelle constitution, cela étoit hor de notre connoissance, et même nous en sommes très punis et sommes tous prest à prendre ceux de la nation $»^{99}$.

En revanche, le maréchal arrêté alors qu'il arbore canne et rubans et se rend d'atelier en atelier pour annoncer son départ, rétorque insolemment

(92) Ibid., 1-D-5, fo 52, 25 juillet 1791.

(93) Ibid., I 2-C 7-D 4, procès-verbal du commissaire Albert.

(94) La rixe des charpentiers est celle qui pousse un maître à écrire pour défendre les compagnons, celle des menuisiers est provoquée par l'irruption de deux compagnons dans un atelier où les outils n'étaient pas rangés (voir plus haut).

(95) AMN, I 2-C 7-D 4, 9 août 1791.

(96) Voir plus haut.

(97) AMN, 1-D-5, fo 101 (citée plus haut).

(98) Ibid., fo 132.

(99) Ibid., I 2-C 7-D 4, pétition des compagnons forgerons, sans date (cette affaire est évoquée par Cynthia Truant, The Rites of Labor, op. cit., p. 201). 
au commissaire de police « que la liberté étoit en France et qu'il n'y a personne dans le cas de les empescher de faire le compagnonage et qu'il existera toujours $»^{100}$. En l'an II, la municipalité sans-culotte, dominée par les maîtres artisans ${ }^{101}$, exige fermement la suppression de la « corporation » des portefaix ${ }^{102}$. Les autorités sont d'autant plus enclines à combattre les organisations ouvrières que, dans le cadre de la mobilisation nationale, elles emploient elles-mêmes de nombreux ouvriers. D'ailleurs, après que la police du travail eut été fortement remise en cause au début de la Révolution, la réquisition constitue sans doute une étape dans sa restauration.

Néanmoins, il faut indiquer qu'au cours de l'hiver 1792-1793, la municipalité et le Commerce jouent les médiateurs entre les ouvriers des différents métiers du port et les maîtres, lorsque ceux-là présentent des pétitions pour obtenir des augmentations de salaire ${ }^{103}$. En l'an II, la municipalité favorise également le compromis salarial, par exemple entre les maîtres et les ouvriers plâtriers ${ }^{104}$. Or, ces démarches n'impliquent-elles pas la reconnaissance implicite des organisations ouvrières à travers l'accueil des délégations et la remise des pétitions ? Il nous semble plus judicieux d'y reconnaître une tolérance imposée par l'acuité des tensions sociales et politiques ${ }^{105}$.

\section{La portée de l'expérience révolutionnaire}

Les organisations ouvrières connaissent-elles des transformations au cours de la Révolution ${ }^{106}$, et, tout d'abord, les ouvriers sont-ils sensibles à l'épanouissement de la sociabilité politique, celle-ci ayant d'ailleurs supplanté de nombreuses formes de sociabilité préexistantes? Les ouvriers essaient peut-être de s'approprier cette nouvelle forme d'association, dans laquelle se fonderaient leur propre effort d'organisation et

(100) ADLA, L 2074, procès-verbal du commissaire Albert, 13 juin 1792.

(101) Issue de la société populaire, la municipalité de l'an II est dominée par les représentants du monde de l'échoppe. Parmi eux, on reconnaît de nombreux maîtres, repérés dans le Calendrier du Commerce, ou le registre des livrets remis aux ouvriers en l'an XII.

(102) AMN, 1-D-9, fo 89, $1^{\text {er }}$ frimaire an II, et F 7-C 7-D 1, 22 thermidor an II.

(103) ADLA, 1 ET-A 7, 8, 12 et 13.

(104) AMN, 1-D-9, fo 93, 23 novembre 1793.

(105) La nécessité de conserver le soutien du peuple en général et des ouvriers en particulier guide l'attitude des autorités, qui peuvent également être sensibles au bien-fondé des revendications. Cependant, tolérance et bienveillance n'empêchent pas les autorités de rester fermes dans leur condamnation de principe d'organisations qu'elles présentent comme des corporations (voir Georges LeFEBVRe, Études orléanaises, t. 2, Subsistances et maximum (1789-an IV), Paris, CHESR, 1963, p. 102 ; Jean-Pierre Gross, Égalitarisme jacobin et droits de l'homme, 1793-1794. La Grande famille et la Terreur, Paris, Arcantères, 2000, p. 340 [1997]).

(106) Steven Kaplan, La fin des corporations, op. cit., p. 451 
leur patriotisme. Une société patriotique apparaît dans une manufacture d'armes ${ }^{107}$. Fondée en 1792 , naît-elle d'une initiative d'ouvriers patriotes? Peut-être donnent-ils en même temps un visage légal à une ancienne organisation ${ }^{108}$. Cependant, cette société pourrait tout aussi bien être suscitée par le patron de la manufacture et les autorités afin de contrôler et de mobiliser les ouvriers engagés dans une si cruciale production. De même que, chez les ouvriers, l'adhésion à l'élan patriotique et le souci de légalisation d'une ancienne organisation ne sont pas incompatibles, de même rien n'interdit d'envisager une initiative commune des autorités, du patron et des ouvriers dans le contexte de la mobilisation pour la défense nationale, même si cela soulève le problème de la genèse de la sans-culotterie en 1791-1792 ${ }^{109}$. Par ailleurs, les ouvriers de l'arsenal de Basse-Indre forment une puissante société qui apporte, en ventôse an III, son soutien à l'un d'eux, sanctionné pour avoir manqué le travail, et, plus généralement, aux revendications ouvrières ${ }^{110}$. Si cette société peut ainsi se manifester au grand jour, c'est peutêtre parce qu'il s'agit d'une société populaire légale ${ }^{111}$. Néanmoins, même si cette société joue le rôle d'une organisation ouvrière traditionnelle et même si ses fondateurs s'efforçaient consciemment de donner une couverture légale à leur organisation, rien ne permet de mettre en doute leur patriotisme, pas plus que la dissimulation d'une organisation clandestine sous le voile d'une confrérie ne permet de douter de la foi des compagnons.

Après que les émeutes populaires, d'une part, et les pétitions ouvrières ${ }^{112}$, de l'autre, ont disparu, des cabales traditionnelles réapparaissent sous le Directoire. Pour autant, faut-il n'y voir que l'indice d'un retour en arrière ou bien ne peut-on tenter d'y déceler l'entretien de l'expérience révolutionnaire des ouvriers nantais ${ }^{113}$ ? Au printemps de l'an V, les ouvriers cordiers s'engagent dans un conflit motivé par une

(107) ADIV, $45 \mathrm{~J} 22$, « association patriotique de la fonderie de Belair ».

(108) Marcel DAVID, Fraternité et Révolution française, 1789-1799, Paris, Aubier, 1987, p. 165.

(109) Voir note 84. Précisons qu'il n'existe pas à Nantes de mouvement sectionnaire.

(110) ADLA, L 2077, pétition de la société des ouvriers de 1'arsenal de Basse-Indre, 21 ventôse an III. Sur les ouvriers de la Navale, voir Norman HAMPSON, « Les ouvriers des arsenaux de la marine au cours de la Révolution française », Revue d'histoire économique et sociale, 1961, p. 287-329 et $442-473$.

(111) Il est surprenant que l'existence de cette société ne soit pas citée, lors des interrogatoires, comme la preuve du « fourvoiement » de ces ouvriers dans un " particularisme égoïste ».

(112) On a signalé notamment la pétition des maçons en 1790 et celles des ouvriers portuaires en 1792-1793.

(113) Pour une nouvelle approche du Directoire, voir Philippe Bourdin, Bernard GaInOT (éd.), La République directoriale, Paris-Clermont-Ferrand, SER-CHEC-CRR, 1998, et Jacques Bernet, Jean-Pierre Jessenne, Hervé Leuwers (éd.), Du Directoire au Consulat, Lille, Université de Valenciennes et CHRENO, 1999-2001. 
revendication salariale. Ils recourent à des pratiques traditionnelles, jetant l'interdit sur plusieurs ateliers et menaçant les récalcitrants. Deux patrons estiment que cette coalition a été « précédé du rétablissement entre eux du cy-devant compagnonnage $»^{114}$. En fait, il est très peu probable que cette organisation ancienne ait disparu au début des années 1790. En revanche, comme le suggère la participation des cordiers au mouvement de pétitions des ouvriers du port en 1792-1793, ils ont peutêtre expérimenté de nouvelles formes de lutte. Par ailleurs, l'organisation dénoncée en l'an $\mathrm{V}$ présente des traits nouveaux. Ainsi, lorsque le juge de paix souligne que « ces rassemblemens sont défendus par la loi puisqu'ils font revivre l'ancien compagnonnage », un ouvrier "répond qu'anciennement on ne soulageait pas ainsi les ouvriers cordiers mais qu'ils veulent aujourd'huy introduire cet usage $\gg^{115}$. Le secours mutuel est attesté par une liste de cotisants. Si l'entraide est ancienne, son institutionnalisation, sous la forme d'une caisse de secours mutuels régulièrement abondée par des cotisations, semble nouvelle. La réapparition de pratiques traditionnelles de lutte et la constitution d'une caisse dans laquelle les maîtres ont pu voir une caisse de coalition, peuvent expliquer que ceux-ci et les autorités aient cru à la renaissance du compagnonnage. En fait, celui-ci n'ayant pas disparu, cette procédure révèle probablement plutôt le développement de sa fonction d'entraide à travers l'institutionnalisation du secours mutuel. Or, ce développement résulte peut-être de l'exaltation de la fraternité au cours de la Révolution et de la prise en charge de cette valeur par l'organisation traditionnelle de la solidarité ouvrière.

Le 3 fructidor an V, le compagnonnage des serruriers est visé par une descente de police. Selon le juge de paix, ces ouvriers « avaient entre eux des correspondances, des registres, des signes et tout ce qui étoit en usage dans les anciennes corporations connues sous le nom de compagnons du devoir $\gg^{116}$. Les compagnons indiquent que leur organisation assure le placement et l'entraide. Lorsque le juge de paix interroge « un citoyen assis, tenant une plume et écrivant des noms sur une feuille [celui-ci répond] que les rassemblements de son état sont tous pour sçavoir s'il y a de l'ouvrage dans les boutiques afin d'y embaucher les arrivants ». La fonction d'entraide semble revêtir une importance nouvelle. Une liste de noms est saisie et un ouvrier précise qu'elle a été rédigée « pour ali-

(114) ADLA, L 2082, plainte d'André Besnier et de Jean Ruelle, 8 prairial an V.

(115) Ibid., interrogatoire de Pierre Luneau, 28 prairial an V.

(116) Ibid., procès-verbal du juge de paix J.-F. Brière. 
menter les indigens et les infirmes parmi les garçons serruriers, en un mot entretenir l'union parmi eux $\gg{ }^{117}$. Il semble donc que les serruriers créent aussi une caisse de secours mutuels. Tandis que la fraternité a pu, dans un premier temps, apparaître aux yeux des ouvriers comme le prolongement révolutionnaire de leur solidarité traditionnelle, il se pourrait qu'ensuite leurs organisations leur permettent de cultiver cette expérience révolutionnaire en favorisant l'épanouissement de cette solidarité ${ }^{118}$.

Dans les années 1800, les autorités déplorent la renaissance d'une « cabale permanente » chez les ouvriers :

«Depuis quelques tems les compagnons recommencent à troubler la tranquillité de cette commune, soit en maltraitant les ouvriers qui ne sont pas du devoir, en allant en trouppe et décorés de rubans par les rues, en forçant les maîtres de n'employer que des compagnons et défendant les boutiques de ceux qui ne se conforment point à leurs prétentions arbitraires $\gg{ }^{119}$.

Plusieurs métiers défendent violemment leur monopole. Les portefaix de la Fosse qui s'emparent de force d'une décharge en vertu de leur monopole sur ce quai, fondent «leurs prétentions sur ce que, disaient-ils, tous les anciens usages se rétablissaient $\rangle^{120}$. Les autorités estiment que les compagnons renouent avec le contrôle de l'embauche. Son abandon est cependant tout aussi douteux que la disparition des compagnonnages. Cette impression de résurgence tient sans doute plutôt à la vigueur de l'opposition ouvrière à la restauration de la police du travail, à travers la réintroduction du livret en 1803 qui s'accompagne à Nantes de la création de plusieurs bureaux de placement ${ }^{121}$.

La résurgence de la « cabale permanente » ne peut être interprétée comme un simple retour à un «Ancien Régime compagnonnique » car les organisations ouvrières des années 1800 présentent des traits nouveaux. Engagée dès la fin de l'Ancien Régime, leur institutionnalisation s'accentue. D'une part, l'esprit de corps s'aiguise si bien que les rixes se multiplient.

(117) Ibid., interrogatoire de Louis Blanchardin, 5 fructidor an V.

(118) Jacques RougerIE, « Le mouvement associatif populaire comme facteur d'acculturation politique à Paris de la Révolution aux années 1840. Continuité, discontinuités », Annales historiques de la Révolution française, 1994-3, p. 503.

(119) ADLA, 1 M 2307, lettre du commissaire Guillet au préfet, 14 floréal an XI.

(120) Ibid., rapport du commissaire Guillet au préfet, 6 fructidor an X.

(121) Ibid., état des bureaux de placement, an XIV. 
Un commissaire note que " les ouvriers de tout états ce rassemblent et parcourent la ville armés de bâtons ; ils y semment la terreur par leurs orgis et batteries [...] toujours pour cause de compagnonage $\gg{ }^{122}$. D'autre part, le développement du secours mutuel se poursuit. Le 4 fructidor an $X$, la police surgit dans une assemblée de cloutiers, occupés à traiter les affaires de secours mutuels. Les règlements d'une société sont saisis : leur rédaction témoigne d'un progrès de l'institutionnalisation du secours mutuel ${ }^{123}$.

Il nous semble donc que l'étude des organisations ouvrières durant la Révolution française exige un élargissement chronologique dans la mesure où la décennie révolutionnaire s'inscrit dans une période plus longue de renforcement et d'évolution de ces organisations au tournant des XVIII et $\mathrm{XIX}^{\mathrm{e}}$ siècles. De plus, cette étude tend à montrer que l'expérience révolutionnaire des ouvriers nantais associe intimement le social et le politique et que, de ce fait, leur participation à la Révolution est, pour partie, façonnée par des motifs originaux. Dès lors, nous proposons de définir la conscience révolutionnaire qui mûrit ${ }^{124}$ chez eux comme l'émergence progressive d'un ensemble de pratiques, de valeurs et de références issues - comme nous l'avions suggéré - tant de leur identité socio-culturelle que de leur participation à la Révolution. Au-delà, cette étude suggère que leur expérience révolutionnaire contribue au façonnement du monde ouvrier confronté à la première industrialisation ${ }^{125}$.

\section{Samuel Guicheteau}

Docteur en histoire, agrégé d'histoire Chercheur associé au Cerhio-Rennes 2

14 rue Jean Bart, 44220 Couëron samuel.guicheteau@orange.fr

(122) Ibid., 7 U 24, rapport du commissaire Durand, 2e jour complémentaire an XII.

(123) Ibid., 7 U 18. Stipulant qu' « il ne viendra que trois bouteille de vin à la fois » lors des assemblées et que, « lorce que le premier ora mis son chapau bas, il commanderas de faire silance », ces règlements suggèrent en outre l'apparition d'une culture de la « décence » (Jacques RougERIE, « Le mouvement associatif... », art. cit., p. 497).

(124) Pour le concept de maturation, voir Michel Vovelle, La mentalité révolutionnaire. Société et mentalités sous la Révolution française, Paris, Messidor-éd. sociales, 1985.

(125) H. Burstin estime que « [la Révolution] va jouer [un rôle] dans le patrimoine historique du radicalisme et en général dans la mémoire historique de la classe ouvrière au XIX ${ }^{\mathrm{e}}$ siècle » (« Pour une histoire socio-politique du Paris révolutionnaire : réflexions méthodologiques », Annales historiques de la Révolution française, 1986-1, p. 34). Voir aussi W. SEWELL, Gens de métier, op. cit. Notre thèse est consacrée à cette question : La Révolution des ouvriers nantais : mutation économique, identité sociale et dynamique révolutionnaire (1740-1815), Rennes, PUR, 2008. 